\title{
Cyclotomic Completions of Polynomial Rings ${ }^{\dagger}$
}

By

\author{
Kazuo HABIRO*
}

\begin{abstract}
For a subset $S \subset \mathbb{N}=\{1,2, \ldots\}$ and a commutative ring $R$ with unit, let $R[q]^{S}$ denote the completion $\varliminf_{f(q)} R[q] /(f(q))$, where $f(q)$ runs over all the products of the powers of cyclotomic polynomials $\Phi_{n}(q)$ with $n \in S$. We will show that under certain conditions the completion $R[q]^{S}$ can be regarded as a "ring of analytic functions" defined on the set of roots of unity of order in $S$. This means that an element of $R[q]^{S}$ vanishes if it vanishes on a certain type of infinite set of roots of unity, or if its power series expansion at one root of unity vanishes. In particular, the completion $\mathbb{Z}[q]^{\mathbb{N}} \simeq \varliminf_{n} \mathbb{Z}[q] /\left((1-q)\left(1-q^{2}\right) \cdots\left(1-q^{n}\right)\right)$ enjoys this property.
\end{abstract}

\section{$\S 1$. Introduction}

For $n \in \mathbb{N}=\{1,2, \ldots\}$, let $\Phi_{n}(q) \in \mathbb{Z}[q]$ denote the $n$th cyclotomic polynomial. Let $S$ be a subset of $\mathbb{N}$. Set $\Phi_{S}=\left\{\Phi_{n}(q) \mid n \in S\right\} \subset \mathbb{Z}[q]$, and let $\Phi_{S}^{*}$ denote the multiplicative set in $\mathbb{Z}[q]$ generated by $\Phi_{S}$. Let $R$ be a commutative ring with unit. The principal ideals $(f(q)) \subset R[q]$ for $f(q) \in \Phi_{S}^{*}$ define a linear topology of the ring $R[q]$. Define a completion $R[q]^{S}$ of $R[q]$ by

$$
R[q]^{S}=\lim _{f\left(\overleftarrow{q) \in \Phi_{S}^{*}}\right.} R[q] /(f(q))
$$

which we will call the $S$-cyclotomic completion of $R[q]$. If $S$ is finite, then $R[q]^{S}$ is just the $\left(\prod_{n \in S} \Phi_{n}(q)\right)$-adic completion of $R[q]$.

Communicated by T. Kawai. Received October 29, 2003. Revised March 1, 2004, June 21, 2004, July 8, 2004.

2000 Mathematics Subject Classification(s): Primary 13B35; Secondary 13B25, 57M27

Key words: completion of polynomial ring, cyclotomic polynomial, Witten-ReshetikhinTuraev invariant

${ }^{\dagger}$ This article is an invited contribution to a special issue of Publications of RIMS commemorating the fortieth anniversary of the founding of the Research Institute for Mathematical Sciences.

* Research Institute for Mathematical Sciences, Kyoto University, Kyoto 606-8502, Japan. 
The main results of this paper can be rephrased as follows: Under certain conditions, the ring $R[q]^{S}$ behaves like a "ring of analytic functions" defined on the set of the roots of unity of order contained in $S$. In the following two paragraphs, we will explain two properties that justify the above claim, by restricting to the special case $R=\mathbb{Z}$ and $S=\mathbb{N}$.

The first property states that an element $f(q) \in \mathbb{Z}[q]^{\mathbb{N}}$ is a function on the set of all the roots of unity. Let $Z_{\mathbb{N}} \subset \mathbb{C}$ denote the subset of all roots of unity, and let $\mathbb{Z}\left[Z_{\mathbb{N}}\right]$ denote the subring of $\mathbb{C}$ generated by the elements of $Z_{\mathbb{N}}$. If $f(q) \in \mathbb{Z}[q]^{\mathbb{N}}$ and $\zeta \in Z_{\mathbb{N}}$, then the evaluation $f(\zeta)$ of $f(q)$ at $\zeta$ is well defined, since $q-\zeta$ divides $\Phi_{n}(q)$ with $n=$ ord $\zeta$. Hence there is a well defined map

$$
\epsilon: \mathbb{Z}[q]^{\mathbb{N}} \rightarrow \operatorname{Map}\left(Z_{\mathbb{N}}, \mathbb{Z}\left[Z_{\mathbb{N}}\right]\right)
$$

such that $\epsilon(f(q))=(f(\zeta))_{\zeta \in Z_{\mathbb{N}}}$. By Theorem 6.2, the map $\epsilon$ is injective, and we can regard $\mathbb{Z}[q]^{\mathbb{N}}$ as a subring of $\operatorname{Map}\left(Z_{\mathbb{N}}, \mathbb{Z}\left[Z_{\mathbb{N}}\right]\right)$. Hence the elements of $\mathbb{Z}[q]^{\mathbb{N}}$ can be regarded as functions defined on the roots of unity. Moreover, Theorem 6.2 implies for example that $f(q) \in \mathbb{Z}[q]^{\mathbb{N}}$ vanishes if $f(q)$ vanishes at infinitely many roots of unity of prime power order.

The second property is a kind of analytic continuation. For $\zeta$ each root of unity, there is an expansion homomorphism

$$
\sigma_{\zeta}: \mathbb{Z}[q]^{\mathbb{N}} \rightarrow \mathbb{Z}[\zeta][[q-\zeta]],
$$

induced by $\mathbb{Z}[q] \rightarrow \mathbb{Z}[\zeta][q]$, since $(q-\zeta)^{i}$ divides $\Phi_{\text {ord } \zeta}(q)^{i}$ for $i \geq 0$. For $f(q) \in \mathbb{Z}[q]^{\mathbb{N}}, \sigma_{\zeta}(f(q))$ can be regarded as the power series expansion of $f(q)$ at $\zeta$. By Theorem 5.2, the homomorphism $\sigma_{\zeta}$ is injective. In other words, the function $\epsilon(f(q))$ is completely determined by its expansion at each root of unity. We remark here that the injectivity of $\sigma_{1}$ is also proved independently by P. Vogel. The non-surjectivity of $\sigma_{\zeta}$ is proved in Section 7.4.

The above-mentioned properties do not hold for a general ring $R$. For example, the analogues of the homomorphisms $\epsilon$ and $\sigma_{\zeta}$ over the rational numbers, are not injective; nevertheless, the natural homomorphism $\mathbb{Z}[q]^{\mathbb{N}} \rightarrow \mathbb{Q}[q]^{\mathbb{N}}$ is injective. For more details, see Section 7.5.

Here we would like to explain the original motivation of studying the cyclotomic completions. We should note first that some specific elements of $\mathbb{Z}[q]^{\mathbb{N}}$ have already appeared in the literature. Zagier [16] studied the series $\sum_{n \geq 0}(1-q)\left(1-q^{2}\right) \cdots\left(1-q^{n}\right)$, which was introduced by Kontsevich, and which can be regarded as an element of $\mathbb{Z}[q]^{\mathbb{N}}$ since we have an isomorphism

$$
\mathbb{Z}[q]^{\mathbb{N}} \simeq \lim _{n} \mathbb{Z}[q] /\left((1-q)\left(1-q^{2}\right) \cdots\left(1-q^{n}\right)\right)
$$


induced by $\mathrm{id}_{\mathbb{Z}[q]}$. Lawrence and Zagier [6] and Le [7] gave formulas for the $s l_{2}$ Witten-Reshetikhin-Turaev invariants $[12,15]$ for some particular integral homology spheres. These formulas were expressed as infinite series which can define elements of $\mathbb{Z}[q]^{\mathbb{N}}$.

The ring $\mathbb{Z}[q]^{\mathbb{N}}$ is used in the definition of the new invariant $I(M)$ of an integral homology 3 -sphere $M$ that we announced in $[1]$ (where $\mathbb{Z}[q]^{\mathbb{N}}$ is denoted by $\widehat{\mathbb{Z}[q]})$, see also [11]. The invariant $I(M)$ takes values in $\mathbb{Z}[q]^{\mathbb{N}}$ and unifies all the Witten-Reshetikhin-Turaev invariants $\tau_{\zeta}(M)$ defined at all the roots of unity $\zeta$, i.e., we have

$$
\epsilon_{\zeta}(I(M))=\tau_{\zeta}(M) \in \mathbb{Z}[\zeta], \quad \text { for all } \zeta \in Z_{\mathbb{N}} .
$$

We may regard this result as saying that the Witten-Reshetikhin-Turaev invariants of an integral homology sphere, viewed as functions on roots of unity, is "analytic". (We note here that Lawrence $[4,5]$ have studied another kind of analyticity of the Witten-Reshetikhin-Turaev invariants.)

As we explained in [1], the existence of the invariant $I(M)$ generalizes the previous integrality results $[9,10,4,13]$ on the Witten-Reshetikhin-Turaev invariants of integral homology spheres. Using the injectivity of $\sigma_{1}: \mathbb{Z}[q]^{\mathbb{N}} \rightarrow$ $\mathbb{Z}[[q-1]]$, we can show that the Ohtsuki series $\tau(M) \in \mathbb{Z}[[q-1]][10]$, which was defined using only the $\tau_{\zeta}(M)$ with $\zeta$ the prime order roots of unity, determine the $\tau_{\zeta}(M)$ for $\zeta$ all the roots of unity. Recall that $\tau(M)$ can be regarded as a kind of "number theoretic expansion" at $q=1$ of the Witten-ReshetikhinTuraev invariants. For $\zeta$ a root of unity, the power series expansion $\epsilon_{\zeta}(I(M)) \in$ $\mathbb{Z}[\zeta][[q-\zeta]]$ in $q-\zeta$ can be regarded as the "number theoretic expansion" at $q=\zeta$ of the Witten-Reshetikhin-Turaev invariants.

The present paper was at first intended to provide the results on the ring $\mathbb{Z}[q]^{\mathbb{N}}$ announced in [1] and those necessary for [2] in which we study completions of an integral form of the quantized enveloping algebra $U_{q}\left(s l_{2}\right)$, and for future papers [3] in which we will prove the existence of the invariant $I(M)$. However, we have generalized the subject of the paper mainly from purely algebraic point of view. Another practical reason for generalization is that it may be possible to define a generalization of $I(M)$ to rational homology spheres with values in $R[q]^{S}$ for some $R$ and $S$ which depend on the first homology group of $M$.

\section{$\S 2 . \quad$ Preliminaries}

Throughout the paper, rings are unital and commutative, and homomorphisms of rings are unital. By "homomorphism" we will usually mean a ring homomorphism. Two rings that are considered to be canonically isomorphic to 
each other will often be identified. Moreover, if a ring $R$ embeds into another ring $R^{\prime}$ in a natural way, we will often regard $R$ as a subring of $R^{\prime}$.

If $R$ is a ring and $I \subset R$ is an ideal, then the $I$-adic completion of $R$ will be denoted by

$$
R^{I}=\lim _{j} R / I^{j}
$$

and if $J \subset I$ is another ideal, then let

$$
\rho_{J, I}^{R}: R^{J} \rightarrow R^{I}
$$

denote the homomorphism induced by $\mathrm{id}_{R}$. The notation $R^{I}$ should not cause confusions with $R[q]^{S}$. We will further generalize these notations in the later sections. The ring $R$ is said to be $I$-adically separated (resp. I-adically complete) if the natural homomorphism $R \rightarrow R^{I}$ is injective (resp. an isomorphism). Recall that $R$ is $I$-adically separated if and only if $\bigcap_{j \geq 0} I^{j}=(0)$.

Let $\mathbb{N}=\{1,2, \ldots\}$ denote the set of positive integers. We regard $\mathbb{N}$ as a directed set with respect to the divisibility relation $\mid$. We will not use the letter $\mathbb{N}$ for the same set $\{1,2, \ldots\}$ when it is considered as an ordered set with the usual order $\leq$.

The letter $q$ will always denote an indeterminate.

\section{§3. Monic Completions of Polynomial Rings}

\section{$\S 3.1$ Definitions and basic properties}

For a ring $R$, let $\mathcal{M}_{R}$ denote the set of the monic polynomials in $R[q]$, which is a directed set with respect to the divisibility relation $\mid$. For a subset $M \subset \mathcal{M}_{R}$, let $M^{*}$ denote the multiplicative set in $R[q]$ generated by $M$, which is a directed subset of $\mathcal{M}_{R}$. The principal ideals $(f), f \in M^{*}$, define a linear topology of the ring $R[q]$, and let

$$
R[q]^{M}=\lim _{f \overleftarrow{\epsilon} M^{*}} R[q] /(f)
$$

denote the completion. (If $M=\{1\}$, then (3.1) implies $R[q]^{\{1\}}=R[q] /(1)=0$, which notationally contradicts the previous definition $R[q]^{\{1\}}=R[[q-1]]$. In the rest of the paper, however, " $R[q]^{\{1\}}$ " will always mean $R[[q-1]]$.)

If $M^{\prime} \subset M \subset \mathcal{M}_{R}$, then $\left(M^{\prime}\right)^{*}$ is a directed subset of $M^{*}$, and hence id $R[q]$ induces a homomorphism

$$
\rho_{M, M^{\prime}}^{R}: R[q]^{M} \rightarrow R[q]^{M^{\prime}} .
$$


We also extend the notation in the obvious way to $\rho_{M, I}^{R}: R[q]^{M} \rightarrow R[q]^{I}$ for $M \subset \mathcal{M}_{R}$ a subset and $I \subset R$ an ideal, etc., if it is well defined. (The general rule is that $\rho_{X, Y}^{R}: R[q]^{X} \rightarrow R[q]^{Y}$ is a homomorphism induced by $\operatorname{id}_{R[q]}$.)

If $M \subset \mathcal{M}_{R}$ is finite, then the sequence $\left(\prod M\right)^{j}, j \geq 0$, is cofinal in the directed set $M^{*}$. Hence $R[q]^{M}$ is naturally isomorphic to the ( $\prod M$ )-adic completion $R[q]^{\left(\prod M\right)}$ of $R[q]$. In particular, if $f \in \mathcal{M}_{R}$, then we have

$$
R[q]^{\{f\}} \simeq R[q]^{(f)}=\lim _{j} R[q] /(f)^{j} .
$$

If $M \subset \mathcal{M}_{R}$ is infinite, then $R[q]^{M}$ is not an ideal-adic completion in general, see for example Proposition 6.1.

If $M \subset \mathcal{M}_{R}$, then the rings $R[q]^{M^{\prime}}$ for finite subsets $M^{\prime}$ of $M$ and the natural homomorphisms $\rho_{M^{\prime}, M^{\prime \prime}}^{R}$ for finite $M^{\prime}, M^{\prime \prime}$ with $M^{\prime \prime} \subset M^{\prime} \subset M$ form an inverse system of rings, of which the inverse limit is naturally isomorphic to $R[q]^{M}$; i.e., we have

$$
R[q]^{M} \simeq \lim _{M^{\prime} \subset M,\left|M^{\prime}\right|<\infty} R[q]^{M^{\prime}} .
$$

Let $h: R \rightarrow R^{\prime}$ be a ring homomorphism. Note that if $h$ is injective (resp. surjective), then so is the induced homomorphism $h_{q}: R[q] \rightarrow R^{\prime}[q]$.

Lemma 3.1. Let $h: R \rightarrow R^{\prime}$ be a ring homomorphism and let $M \subset$ $\mathcal{M}_{R}$ be a subset. If $h$ is injective, then so is the homomorphism

$$
h_{M}: R[q]^{M} \rightarrow R^{\prime}[q]^{h(M)}
$$

induced by $h_{q}$. If $h$ is surjective and $M$ is at most countable, then $h_{M}$ is surjective.

Proof. For each $f \in M^{*}$, the $R$-module $R[q] /(f)$ is free of $\operatorname{rank} \operatorname{deg} f$, since $f$ is a monic polynomial. If $h$ is injective, then the natural homomorphism

$$
h_{f}: R[q] /(f) \rightarrow R[q] /(f) \otimes_{R} R^{\prime}=R^{\prime}[q] /(h(f))
$$

is injective. Taking the inverse limit, we see that the induced map $h_{M}$ is injective.

Suppose $h$ is surjective and $M$ is at most countable. There is a sequence $g_{0}\left|g_{1}\right| \cdots$ in $M^{*}$ which is cofinal in $M^{*}$. Since the topology of $R^{\prime}[q]$ defined by the $\left(h\left(g_{n}\right)\right)$ is induced along the surjective homomorphism $h_{q}: R[q] \rightarrow R^{\prime}[q]$ by the topology of $R[q]$ defined by the $\left(g_{n}\right)$, it follows that $h_{M}$ is surjective. (See, e.g., $[8$, Theorem 8.1. (ii)].) 


\section{§3.2. Injectivity of the homomorphism $\rho_{M, M^{\prime}}^{R}$}

Let $R$ be a ring, $I \subset R$ an ideal, and $f, g \in \mathcal{M}_{R}$. Let $\sqrt{I}$ denote the radical of $I$. We write $f \stackrel{I}{\Rightarrow} R$, or simply $f \stackrel{I}{\Rightarrow} g$, if $f \in \sqrt{(g)+I[q]}$, i.e., if $f^{m} \in(g)+I[q]$ for some $m \geq 0$. For $f, g \in \mathcal{M}_{R}$, we write $f \Rightarrow_{R} g$, or simply $f \Rightarrow g$, if we have $f \stackrel{I}{\Rightarrow} R$ g for some ideal $I \subset R$ with $\bigcap_{j \geq 0} I^{j}=(0)$. Then $\Rightarrow_{R}$ defines a relation on the set $\mathcal{M}_{R}$. Obviously, $g \mid f$ implies $f \Rightarrow g$. Note also that if $f \Rightarrow g, f \mid f^{\prime}$, and $g^{\prime} \mid g$, then $f^{\prime} \Rightarrow g^{\prime}$.

Proposition 3.1. Let $R$ be a ring, and $f, g \in \mathcal{M}_{R}$ with $f \Rightarrow_{R} g$. Then the homomorphism $\rho_{(f g),(f)}^{R}: R[q]^{(f g)} \rightarrow R[q]^{(f)}$ is injective.

Proof. We first show that if $f \stackrel{I}{\Rightarrow} g$ and $R$ is $I$-adically complete, then $\rho_{(f g),(f)}^{R}$ is an isomorphism. Since $R \simeq R^{I}$ and $f$ is monic, we have

$$
\begin{aligned}
& R[q]^{(f)} \simeq R^{I}[q]^{(f)}=\lim _{i}(\underbrace{\lim }_{j} R / I^{j})[q] /\left(f^{i}\right) \\
& \simeq \lim _{i}\left(\lim _{j} R[q] /\left(\left(f^{i}\right)+I^{j}[q]\right)\right) \simeq R[q]^{(f)+I[q]} .
\end{aligned}
$$

Similarly, $R[q]^{(f g)} \simeq R[q]^{(f g)+I[q]}$. Since $f \stackrel{I}{\Rightarrow} g$, we have $((f)+I[q])^{m} \subset$ $\left(f^{m}\right)+I[q] \subset(f g)+I[q]$ for some $m \geq 1$, while we obviously have $(f g)+I[q] \subset$ $(f)+I[q]$. Hence the $((f)+I[q])$-adic topology and the $((f g)+I[q])$-adic topology of $R[q]$ are the same. Hence $\rho_{(f g)+I[q],(f)+I[q]}^{R}$, which may be identified with $\rho_{(f g),(f)}^{R}$, is an isomorphism.

Now consider the general case, where we have $f \stackrel{I}{\Rightarrow}_{R} g$ and $R$ is $I$-adically separated. We have a commutative diagram

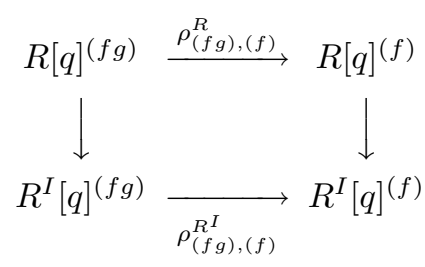

where vertical arrows are induced by the inclusion $R \subset R^{I}$, and hence are injective. Let $\bar{I}$ denote the closure of $I$ in $R^{I}$. Since $R^{I}$ is $\bar{I}$-adically complete and clearly $f \stackrel{\bar{I}}{\Rightarrow} R^{I} g$, the above-proved case implies that $\rho_{(f g),(f)}^{R^{I}}$ is an isomorphism. Hence $\rho_{(f g),(f)}^{R}$ is injective.

For two subsets $M, M^{\prime} \subset \mathcal{M}_{R}$, we write $M^{\prime} \prec M$ if $M^{\prime} \subset M$ and for each $f \in M$ there is a sequence $M^{\prime} \ni f_{0} \Rightarrow f_{1} \Rightarrow \cdots \Rightarrow f_{r}=f$ in $M$. 
Suppose that $M_{0} \prec M \subset \mathcal{M}_{R}$. Set

$$
\mathcal{F}\left(M, M_{0}\right)=\left\{M^{\prime} \subset M\left|M_{0} \subset M^{\prime},\right| M^{\prime} \backslash M_{0} \mid<\infty\right\},
$$

and

$$
\mathcal{F}^{\prec}\left(M, M_{0}\right)=\left\{M^{\prime} \in \mathcal{F}\left(M, M_{0}\right) \mid M_{0} \prec M^{\prime}\right\} \subset \mathcal{F}\left(M, M_{0}\right) .
$$

We will regard $\mathcal{F}\left(M, M_{0}\right)$ as a directed set with respect to $\subset$, and $\mathcal{F}^{\prec}\left(M, M_{0}\right)$ as a partially-ordered subset of $\mathcal{F}\left(M, M_{0}\right)$. Note that if $M^{\prime}, M^{\prime \prime} \in \mathcal{F}^{\prec}\left(M, M_{0}\right)$ and $M^{\prime \prime} \subset M^{\prime}$, then we have $M^{\prime \prime} \prec M^{\prime}$.

Lemma 3.2. If $M_{0} \prec M \subset \mathcal{M}_{R}$, then $\mathcal{F}^{\prec}\left(M, M_{0}\right)$ is a cofinal directed subset of $\mathcal{F}\left(M, M_{0}\right)$.

Proof. It suffices to show that if $M^{\prime} \in \mathcal{F}\left(M, M_{0}\right)$, then there is $M^{\prime \prime} \in$ $\mathcal{F}^{\prec}\left(M, M_{0}\right)$ with $M^{\prime} \subset M^{\prime \prime}$. For each $g \in M^{\prime} \backslash M_{0}$, choose a sequence $M_{0} \ni g_{0} \Rightarrow \cdots \Rightarrow g_{r}=g$ in $M$ and set $U_{g}=\left\{g_{1}, \ldots, g_{r}\right\}$. Set $M^{\prime \prime}=$ $M_{0} \cup \bigcup_{g \in M^{\prime} \backslash M_{0}} U_{g}$. Then we have $M^{\prime \prime} \in \mathcal{F}^{\prec}\left(M, M_{0}\right)$ and $M^{\prime} \subset M^{\prime \prime}$.

Theorem 3.1. If $R$ is a ring and $M_{0} \prec M \subset \mathcal{M}_{R}$, then the homomorphism $\rho_{M, M_{0}}^{R}: R[q]^{M} \rightarrow R[q]^{M_{0}}$ is injective.

Proof. By (3.2) and Lemma 3.2 we have

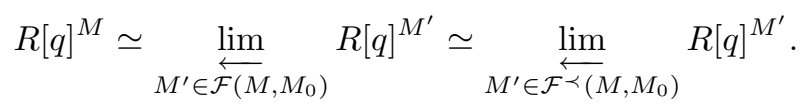

Hence it suffices to prove the theorem assuming that $M \backslash M_{0}$ is finite. We can further assume that $\left|M \backslash M_{0}\right|=1$. Let $g \in M \backslash M_{0}$ be the unique element.

First we assume that $M_{0}=\left\{f_{1}, \ldots, f_{n}\right\}(n \geq 1)$ is finite. Set $f=f_{1} \cdots f_{n}$. Since $f_{i} \Rightarrow g$ for some $i \in\{1, \ldots, n\}$, we have $f \Rightarrow g$. By Proposition 3.1, $\rho_{(f g),(f)}^{R}$ is injective. Since $R[q]^{M_{0}}=R[q]^{(f)}$ and $R[q]^{M}=R[q]^{(f g)}$, it follows that $\rho_{M, M_{0}}^{R}$ is injective.

Now assume that $M_{0}$ is infinite. Choose an element $g_{0} \in M_{0}$ with $g_{0} \Rightarrow g$. We have $R[q]^{M_{0}} \simeq \varliminf_{U \in \mathcal{F}\left(M_{0},\left\{g_{0}\right\}\right)} R[q]^{U}$ and $R[q]^{M} \simeq \varliminf_{U \in \mathcal{F}\left(M_{0},\left\{g_{0}\right\}\right)} R[q]^{U \cup\{g\}}$. For each $U \in \mathcal{F}\left(M_{0},\left\{g_{0}\right\}\right)$, we have $U \prec U \cup\{g\}$. Hence it follows from the above-proved case that the homomorphism $\rho_{U \cup\{g\}, U}^{R}: R[q]^{U \cup\{g\}} \rightarrow R[q]^{U}$ is injective. Since $\rho_{M, M_{0}}^{R}$ is the inverse limit of the $\rho_{U \cup\{g\}, U}^{R}$ for $U \in \mathcal{F}\left(M_{0},\left\{g_{0}\right\}\right)$, it is injective.

A subset $M \subset \mathcal{M}_{R}$ is said to be $\Rightarrow_{R}$-connected if $M$ is not empty and for each $f, f^{\prime} \in M$ there is a sequence $f=f_{0} \Rightarrow_{R} f_{1} \Rightarrow_{R} \cdots \Rightarrow_{R} f_{r}=f^{\prime}(r \geq 0)$ in 
$M$. Note that if $M$ is $\Rightarrow_{R}$-connected, then for any nonempty subset $M^{\prime} \subset M$ we have $M^{\prime} \prec M$. The following follows immediately from Theorem 3.1.

Corollary 3.1. If $R$ is a ring, and $M \subset \mathcal{M}_{R}$ is a $\Rightarrow_{R}$-connected subset, then for any nonempty subset $M^{\prime} \subset M$ the homomorphism $\rho_{M, M^{\prime}}^{R}: R[q]^{M} \rightarrow$ $R[q]^{M^{\prime}}$ is injective.

\section{$\S 4 . \quad$ Injectivity of $\rho_{S, S^{\prime}}^{R}$}

If $R$ a ring, and $S \subset \mathbb{N}$ is a subset, then the completion $R[q]^{S}$ defined in the introduction can be identified with $R[q]^{\Phi_{S}}$. If $S^{\prime} \subset S$, then we set

$$
\rho_{S, S^{\prime}}^{R}=\rho_{\Phi_{S}, \Phi_{S^{\prime}}}^{R}: R[q]^{S} \rightarrow R[q]^{S^{\prime}} .
$$

In this section, we will study injectivity of $\rho_{S, S^{\prime}}^{R}$.

We will use the following well-known properties of cyclotomic polynomials.

Lemma 4.1. (1) Let $n \in \mathbb{N}, p$ a prime, and $e \geq 1$. Then we have

$$
\Phi_{p^{e} n}(q) \equiv \Phi_{n}(q)^{d} \quad(\bmod (p)),
$$

in $\mathbb{Z}[q]$, where $d=\operatorname{deg} \Phi_{p^{e} n}(q) / \operatorname{deg} \Phi_{n}(q)$. (We have $d=(p-1) p^{e-1}$ if $(n, p)=$ 1 and $d=p^{e}$ if $p \mid n$.) Also, we have

$$
p \in\left(\Phi_{n}(q), \Phi_{p^{e} n}(q)\right)
$$

in $\mathbb{Z}[q]$.

(2) If $m, n \in \mathbb{N}$, and $n / m \in \mathbb{Q}$ is not an integer power of a prime, then we have $\left(\Phi_{n}(q), \Phi_{m}(q)\right)=(1)$ in $\mathbb{Z}[q]$.

Proof. (4.2) follows from $p \equiv \sum_{i=0}^{p-1} q^{i p^{e-1} n} \bmod \left(\Phi_{n}(q)\right)$, and

$$
\sum_{i=0}^{p-1} q^{i p^{e-1} n}=\frac{q^{p^{e} n}-1}{q^{p^{e-1} n}-1} \in\left(\Phi_{p^{e} n}(q)\right) .
$$

The other assertions are more familiar.

For $m, n \in \mathbb{N}$, we define $c_{m, n} \in\{0,1\} \cup\{p \mid$ prime $\}$ by

1. $c_{n, n}=0$,

2. $c_{m, n}=p$ if $p$ is a prime and $n / m=p^{j}$ for some $j \in \mathbb{Z} \backslash\{0\}$, and 
3. $c_{m, n}=1$ if $n / m$ is not an integer power of a prime.

Note that $c_{m, n}=c_{n, m}$ for all $m, n \in \mathbb{N}$.

For a ring $R \neq\{0\}$, let $\Leftrightarrow_{R}$ denote the binary relation on $\mathbb{N}$ such that, for $m, n \in \mathbb{N}$, we have $m \Leftrightarrow_{R} n$ if and only if $R$ is $\left(c_{m, n}\right)$-adically separated. Note that we have $m \Leftrightarrow_{R} n$ if and only if $n / m$ is either 1 or an integer-power of a prime $p$ such that $R$ is $p$-adically separated. Note also that the binary relation $\Leftrightarrow_{R}$ is reflexive and symmetric, but not transitive in general.

Lemma 4.2. (1) For each $m, n \in \mathbb{N}$ we have $\Phi_{m}(q) \in \sqrt{\left(\Phi_{n}(q), c_{m, n}\right)}$ in $R[q]$, i.e., $\Phi_{m}(q) \stackrel{\left(c_{m, n}\right)}{\Rightarrow}{ }_{R} \Phi_{n}(q)$.

(2) We have $m \Leftrightarrow_{R} n$ if and only if we have $\Phi_{m}(q) \Rightarrow_{R} \Phi_{n}(q)$.

Proof. (1) and the "only if" part of (2) follows easily from Lemma 4.1. We will show the "if" part of (2). The case $c_{m, n}=0$ is obvious, and the case $c_{m, n}=1$ follows easily from Lemma $4.1(2)$.

Suppose that $c_{m, n}=p$ is a prime, and $\Phi_{m}(q) \Rightarrow_{R} \Phi_{n}(q)$ holds. Thus, there is an ideal $I$ in $R$ such that $R$ is $I$-adically separated, and $\Phi_{m}(q)^{i} \in$ $\left(\Phi_{n}(q)\right)+I[q]$ in $R[q]$ for some $i \geq 0$. Hence, by (4.2), we have $p^{i} \in\left(\Phi_{n}(q)\right)+I[q]$ in $R[q]$. Since $\Phi_{n}(q)$ is a monic polynomial, it follows that $p^{i} \in I$. Since $R$ is $I$-adically separated, $R$ is also $p$-adically separated and we have the assertion.

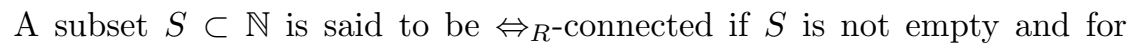
each $n, n^{\prime} \in S$ there is a sequence $n=n_{0} \Leftrightarrow_{R} n_{1} \Leftrightarrow_{R} \cdots \Leftrightarrow_{R} n_{r}=n^{\prime}(r \geq 0)$ in $S$. Note that $S \subset \mathbb{N}$ is $\Leftrightarrow_{R}$-connected if and only if $\Phi_{S}$ is $\Rightarrow_{R}$-connected. The following follows immediately from Theorem 3.1, Corollary 3.1, and Lemma 4.2.

Theorem 4.1. $\quad$ Let $R$ be a ring and let $S^{\prime} \subset S \subset \mathbb{N}$. Suppose that for each element $n \in S$, there is a sequence $S^{\prime} \ni n^{\prime} \Leftrightarrow_{R} \cdots \Leftrightarrow_{R} n$ in $S$. Then the homomorphism $\rho_{S, S^{\prime}}^{R}$ is injective.

In particular, if $S \subset \mathbb{N}$ is $\Leftrightarrow_{R}$-connected, then for any nonempty subset $S^{\prime} \subset S$ the homomorphism $\rho_{S, S^{\prime}}^{R}: R[q]^{S} \rightarrow R[q]^{S^{\prime}}$ is injective. More particularly, for any nonempty subset $S^{\prime} \subset \mathbb{N}$ the homomorphism $\rho_{\mathbb{N}, S^{\prime}}^{\mathbb{Z}}: \mathbb{Z}[q]^{\mathbb{N}} \rightarrow$ $\mathbb{Z}[q]^{S^{\prime}}$ is injective.

We remark that the special case of Theorem 4.1 where $R=\mathbb{Z}, S=\mathbb{N}$, and $S^{\prime}=\{1\}$ is obtained also by P. Vogel. Another proof of a special case of Theorem 4.1 is sketched in Remark 5.1. 
For each $n \in \mathbb{N}$, set $\langle n\rangle=\{m \in \mathbb{N}|m| n\}$. Since $\prod \Phi_{\langle n\rangle}=\prod_{m \mid n} \Phi_{m}(q)=$ $q^{n}-1$, we have

$$
R[q]^{\langle n\rangle}=R[q]^{\left(q^{n}-1\right)}=\lim _{j} R[q] /\left(q^{n}-1\right)^{j} .
$$

Note that the set $\langle n\rangle$ is $\Leftrightarrow_{R}$-connected if and only if for each prime factor $p$ of $n$ the ring $R$ is $p$-adically separated.

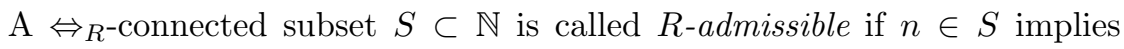
$\langle n\rangle \subset S$, and $a, b \in S$ implies $\exists c \in S$ such that $a|c, b| c$. Note that a subset $S \subset \mathbb{N}$ is finite and $R$-admissible if and only if there is $n \in \mathbb{N}$ such that $S=\langle n\rangle$ and $R$ is $p$-adically separated for each prime factor $p$ of $n$. Note also that an $R$-admissible subset $S \subset \mathbb{N}$ satisfies $S=\bigcup_{n \in S}\langle n\rangle$, and hence we have $R[q]^{S} \simeq \varliminf_{n \in S} R[q]^{\langle n\rangle}$. The following follows easily from Theorem 4.1.

Corollary 4.1. Let $R$ be a ring, and let $S \subset \mathbb{N}$ be $R$-admissible. Then for each $m, n \in S$ with $m \mid n$ the homomorphism $\rho_{\langle n\rangle,\langle m\rangle}^{R}: R[q]^{\langle n\rangle} \rightarrow R[q]^{\langle m\rangle}$ is injective. Hence $R[q]^{S}$ can be regarded as the intersection $\bigcap_{n \in S} R[q]^{\langle n\rangle}$, where the $R[q]^{\langle n\rangle}, n \in S$, are regarded as $R$-subalgebras of $R[q]^{\langle 1\rangle}=R[[q-1]]$.

In particular, if $m, n \in \mathbb{N}$ and $m \mid n$, then $\rho_{\langle n\rangle,\langle m\rangle}^{\mathbb{Z}}: \mathbb{Z}[q]^{\langle n\rangle} \rightarrow \mathbb{Z}[q]^{\langle m\rangle}$ is injective. We have $\mathbb{Z}[q]^{\mathbb{N}}=\bigcap_{n \in \mathbb{N}} \mathbb{Z}[q]^{\langle n\rangle}$.

We will see in Proposition 7.4 that if $m \mid n$ and $m \neq n$, then $\rho_{\langle n\rangle,\langle m\rangle}^{\mathbb{Z}}$ is not surjective.

\section{§5. Expansions at Roots of Unity}

For an integral domain $R$ of characteristic 0 , let $Z^{R}$ denote the set of the roots of unity in $R$. If $S \subset \mathbb{N}$, then set $Z_{S}^{R}=\left\{\zeta \in Z^{R} \mid\right.$ ord $\left.\zeta \in S\right\}$. For a subset $Z \subset Z^{R}$, set

$$
R[q]^{Z}=R[q]^{M_{Z}},
$$

where $M_{Z}=\{q-\zeta \mid \zeta \in Z\} \subset \mathcal{M}_{R}$. If $Z^{\prime} \subset Z$, then set

$$
\rho_{Z, Z^{\prime}}^{R}=\rho_{M_{Z}, M_{Z^{\prime}}}^{R}: R[q]^{Z} \rightarrow R[q]^{Z^{\prime}} .
$$

(Although we have $1 \in Z$ and $1 \in \mathbb{N}$, the notation $R[q]^{\{1\}}$ is not ambiguous because 1 is the unique primitive 1 st root of unity.)

For a subset $Z \subset Z^{R}$, set $N_{Z}=\{\operatorname{ord} \zeta \mid \zeta \in Z\}$, and in particular set $N_{R}=N_{Z^{R}}$. If $S \subset N_{R}$, then we have

$$
R[q]^{S} \simeq R[q]^{Z_{S}^{R}} .
$$

Lemma 5.1. Let $R$ be an integral domain of characteristic 0 , and let $\zeta, \zeta^{\prime} \in Z^{R}$. Then the following conditions are equivalent. 
1. $(q-\zeta) \Rightarrow_{R}\left(q-\zeta^{\prime}\right)$,

2. $R$ is $\left(\zeta-\zeta^{\prime}\right)$-adically separated,

3. $\operatorname{ord}\left(\zeta^{-1} \zeta^{\prime}\right)$ is a power of some prime $p$ such that $R$ is $p$-adically separated.

Proof. If (1) holds, then we have $(q-\zeta)^{m} \in\left(q-\zeta^{\prime}\right)+I[q]$ for some $m \geq 0$ and $R$ is $I$-adically separated. It follows that $\left(\zeta^{\prime}-\zeta\right)^{m} \in I$, and hence $R$ is $\left(\zeta^{\prime}-\zeta\right)$-adically separated. Hence we have $(2)$.

It is straightforward to prove that (2) implies (1), and that (2) and (3) are equivalent.

Let $\Leftrightarrow_{R}$ denote the relation on $Z^{R}$ such that for $\zeta, \zeta^{\prime} \in Z^{R}$ we have $\zeta \Leftrightarrow_{R} \zeta^{\prime}$ if and only if at least one of the conditions in Lemma 5.1 holds. The following theorem follows immediately from Corollary 3.1.

Theorem 5.1. $\quad$ Let $R$ be an integral domain of characteristic 0 and let $Z \subset Z^{R}$ be $a \Leftrightarrow_{R}$-connected subset. Then for any nonempty subset $Z^{\prime} \subset Z$ the homomorphism $\rho_{Z, Z^{\prime}}^{R}: R[q]^{Z} \rightarrow R[q]^{Z^{\prime}}$ is injective.

Lemma 5.2. $\quad$ Let $R$ be an integral domain of characteristic 0 , and $Z \subset$ $Z^{R}$. We have the following.

1. If $Z$ is $\Leftrightarrow_{R}$-connected, then $N_{Z}$ is $\Leftrightarrow_{R}$-connected.

2. Suppose that if $\zeta \in Z, \zeta^{\prime} \in Z^{R}$ and $\operatorname{ord} \zeta=\operatorname{ord} \zeta^{\prime}$, then $\zeta^{\prime} \in Z$. Then if $N_{Z}$ is $\Leftrightarrow_{R}$-connected, then $Z$ is $\Leftrightarrow_{R}$-connected.

Proof. The first assertion follows from the fact that if $\zeta, \zeta^{\prime} \in Z^{R}$, then $\zeta \Leftrightarrow_{R} \zeta^{\prime}$ implies ord $\zeta \Leftrightarrow_{R}$ ord $\zeta^{\prime}$.

The second assertion follows from the fact that if ord $\zeta \Leftrightarrow_{R}$ ord $\zeta^{\prime}$ holds, then we have $\zeta^{a} \Leftrightarrow_{R}\left(\zeta^{\prime}\right)^{a^{\prime}}$ for some $a, a^{\prime} \in \mathbb{Z}$ such that $(a$,ord $\zeta)=1$, $\left(a^{\prime}, \operatorname{ord} \zeta^{\prime}\right)=1$.

Remark 5.1. We sketch below another proof using Theorem 5.1 of the special case of Theorem 4.1 where $S$ is $\Leftrightarrow_{R}$-connected and $R$ is an integral domain of characteristic 0 such that $R$ is $p$-adically separated for any prime $p$. Let $k$ be the quotient field of $R$ and let $\bar{k}$ be the algebraic closure of $k$. Let $\tilde{R} \subset \bar{k}$ be the $R$-subalgebra generated by the elements of $Z_{S}^{\bar{k}}$. In view of Lemma 3.1, it suffices to see that $\rho_{S, S^{\prime}}^{\tilde{R}}$ is injective. Since $S$ is $\Leftrightarrow_{R}$-connected,

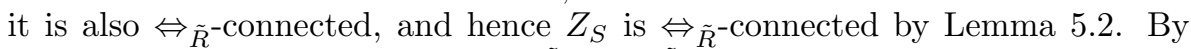
Theorem 5.1, the homomorphism $\rho_{S, S^{\prime}}^{\tilde{R}}=\rho_{Z_{S}, Z_{S^{\prime}}}^{\tilde{R}}$ is injective. 
Theorem 5.2. $\quad$ Let $R$ be an integral domain of characteristic $0, S \subset \mathbb{N}$ $a \Leftrightarrow_{R}$-connected subset, and $n \in S$. Assume that $R$ is p-adically separated for each odd prime factor $p$ of $n$, and also that if $4 \mid n$, then $R$ is 2-adically separated. Let $\zeta$ be a primitive nth root of unity in the algebraic closure of the quotient field of $R$, which may or may not be contained in $R$. Then the homomorphism

$$
\sigma_{S, \zeta}^{R}: R[q]^{S} \rightarrow R[\zeta][[q-\zeta]]
$$

induced by $R[q] \subset R[\zeta][q]$ is injective. (Note that if $\zeta \in R$ then we have $R[\zeta]=$ R.)

In particular, for any root $\zeta$ of unity the homomorphism $\sigma_{\mathbb{N}, \zeta}^{\mathbb{Z}}: \mathbb{Z}[q]^{\mathbb{N}} \rightarrow$ $\mathbb{Z}[\zeta][[q-\zeta]]$ is injective.

Proof. By Lemma 3.1, the homomorphism $R[q]^{S} \rightarrow R[\zeta][q]^{S}$ is injective. Hence we may assume $\zeta \in R$ without loss of generality.

The homomorphism $\sigma_{S, \zeta}^{R}$ is the composition of the following two homomorphisms

$$
R[q]^{S} \stackrel{\rho_{S,\{n\}}^{R}}{\longrightarrow} R[q]^{\{n\}} \stackrel{\rho_{\{n\},(q-\zeta)}^{R}}{\longrightarrow} R[[q-\zeta]] .
$$

The first arrow $\rho_{S,\{n\}}^{R}$ is injective by Theorem 4.1. Hence it suffices to prove that $\rho_{\{n\},(q-\zeta)}^{R}$ is injective.

For each $m$ with $m \mid n$, set $Z_{m}=Z_{\{m\}}^{R}=\left\{\zeta \in Z^{R} \mid\right.$ ord $\left.\zeta=m\right\}$. By $R[q]^{\{n\}} \simeq R[q]^{Z_{n}}$ and Theorem 5.1, it suffices to prove that the set $Z_{n}$ is

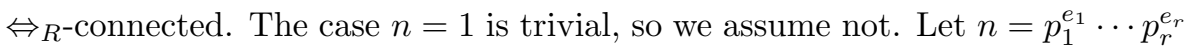
be a factorization into prime powers, where $p_{1}, \ldots, p_{r}$ are distinct primes and $e_{1}, \ldots, e_{r} \geq 1$. There is a bijection

$$
Z_{p_{1}^{e_{1}}} \times \cdots \times Z_{p_{r}^{e_{r}}} \stackrel{\simeq}{\longrightarrow} Z_{n}, \quad\left(\xi_{1}, \ldots, \xi_{r}\right) \mapsto \xi_{1} \cdots \xi_{r} .
$$

It suffices to show that if $\left(\xi_{1}, \ldots, \xi_{r}\right),\left(\xi_{1}^{\prime}, \ldots, \xi_{r}^{\prime}\right) \in Z_{p_{1}^{e_{1}}} \times \cdots \times Z_{p_{r}^{e_{r}}}$ satisfies $\xi_{j}=\xi_{j}^{\prime}$ for all $j \in\{1, \ldots, r\} \backslash\{i\}$ and $\xi_{i} \neq \xi_{i}^{\prime}$ for some $i$, then we have $\xi_{1} \cdots \xi_{r} \Leftrightarrow_{R} \xi_{1}^{\prime} \cdots \xi_{r}^{\prime}$, which is equivalent to that $\xi_{i} \Leftrightarrow_{R} \xi_{i}^{\prime}$. Since $Z_{2}=\{-1\}$ contains only one element, the case $p_{i}=2$ and $e_{i}=1$ does not occur. We have $\left(\xi_{i}-\xi_{i}^{\prime}\right) \subset \sqrt{\left(p_{i}\right)}$, and hence $\xi_{i} \Leftrightarrow_{R} \xi_{i}^{\prime}$.

Corollary 5.1. Let $R$ be an integral domain of characteristic 0 , and $S \subset \mathbb{N} a \Leftrightarrow_{R}$-connected subset. Suppose that there is $n \in S$ such that $R$ is $p$-adically separated for each odd prime factor $p$ of $n$, and if $4 \mid n$, then $R$ is also 2-adically separated. Then the ring $R[q]^{S}$ is an integral domain.

In particular, $\mathbb{Z}[q]^{S}$ is an integral domain for any nonempty subset $S \subset \mathbb{N}$. 
Proof. The result follows from Theorem 5.2 and the fact that the formal power series ring $R[\zeta][[q-\zeta]]$ is an integral domain.

\section{$\S 6 . \quad$ Values at Roots of Unity}

Let $R$ be a subring of the field $\overline{\mathbb{Q}}$ of algebraic numbers and let $S \subset \mathbb{N}$. For $T \subset S$, set

$$
P_{T}(R)=\prod_{n \in T} R[q] /\left(\Phi_{n}(q)\right)
$$

and let

$$
\epsilon_{S, T}^{R}: R[q]^{S} \rightarrow P_{T}(R)
$$

be induced by the homomorphism $R[q] \rightarrow P_{T}(R), f(q) \mapsto(f(q) \bmod$ $\left.\left(\Phi_{n}(q)\right)\right)_{n \in T}$.

Theorem 6.1. Let $R$ be a subring of $\overline{\mathbb{Q}}, S \subset \mathbb{N} a \Leftrightarrow_{R}$-connected subset, and $T \subset S$ a subset. Suppose that for some $n \in S$ there are infinitely many elements $m \in T$ with $m \Leftrightarrow_{R} n$. Then the homomorphism $\epsilon_{S, T}^{R}: R[q]^{S} \rightarrow P_{T}(R)$ is injective.

In particular, if $R$ is a subring of the ring of algebraic integers, then, for any subset $T \subset \mathbb{N}$ containing infinitely many prime powers, $\epsilon_{\mathbb{N}, T}^{R}: R[q]^{\mathbb{N}} \rightarrow P_{T}(R)$ is injective.

Proof. Suppose to the contrary that there is a nonzero element $a \in R[q]^{S}$ with $\epsilon_{S, T}^{R}(a)=0$. By Theorem 4.1, $\rho_{S,\{n\}}^{R}$ is injective, and therefore we have $\rho_{S,\{n\}}^{R}(a) \neq 0$. Hence we can write $\rho_{S,\{n\}}^{R}(a)=\sum_{j=l}^{\infty} a_{j} \Phi_{n}(q)^{j}$, where $l \geq 0$ and $a_{j} \in R[q]$ for $j \geq l$ with $a_{l} \notin\left(\Phi_{n}(q)\right)$.

Now observe that there are infinitely many elements $m_{1}, m_{2}, \ldots \in T$ with $m_{i} \Leftrightarrow_{R} n$ and $n \mid m_{i}$. For each $i, m_{i} / n$ is a power of a prime $p_{i}$ such that $R$ is $p_{i}$-adically separated. It follows from $\epsilon_{S, T}^{R}(a)=0$ that $\Phi_{m_{i}}(q) \mid a$ in $R[q]^{S}$ for each $i$.

We claim that we have $\Phi_{m_{1}}(q) \cdots \Phi_{m_{k}}(q) \mid a$ in $R[q]^{S}$ for each $k \geq 0$. We will prove this claim by induction on $k$. Since the case $k=0$ is trivial, suppose $k \geq 1$. By assumption, we have $\Phi_{m_{1}}(q) \cdots \Phi_{m_{k-1}}(q) \mid a$ in $R[q]^{S}$. Since $m_{k} \in S$, there are $b(q) \in R[q]$ and $c \in R[q]^{S}$ such that

$$
a=\Phi_{m_{1}}(q) \cdots \Phi_{m_{k-1}}(q)\left(b(q)+\Phi_{m_{k}}(q) c\right) .
$$


Since $\Phi_{m_{k}} \mid a$, we have $\Phi_{m_{k}}(q) \mid \Phi_{m_{1}}(q) \cdots \Phi_{m_{k-1}}(q) b(q)$ in $R[q]^{S}$. Hence we have

$$
\Phi_{m_{1}}\left(\zeta_{m_{k}}\right) \cdots \Phi_{m_{k-1}}\left(\zeta_{m_{k}}\right) b\left(\zeta_{m_{k}}\right)=0
$$

in $R$. Since $\Phi_{m_{j}}\left(\zeta_{m_{k}}\right) \neq 0$ for $j=1, \ldots, k-1$, it follows that $b\left(\zeta_{m_{k}}\right)=0$, and hence $\Phi_{m_{k}}(q) \mid b(q)$. By (6.1), we obtain the claim.

It follows from the above claim that we have $\Phi_{m_{1}}(q) \cdots \Phi_{m_{k}}(q) \mid \rho_{S,\{n\}}^{R}(a)$ in $R[q]^{\{n\}}$. By (4.1) we have $\Phi_{m_{i}}(q) \in\left(p_{i}, \Phi_{n}(q)\right)$ for each $i$. Hence we have $\Phi_{m_{1}}(q) \cdots \Phi_{m_{k}}(q) \in\left(p_{1} \cdots p_{k}, \Phi_{n}(q)\right)$. In other words, for each $k \geq 0, \bar{a}_{l}=a_{l}$ $\bmod \left(\Phi_{n}(q)\right) \in R[q] /\left(\Phi_{n}(q)\right)$ is divisible by $p_{1} \cdots p_{k}$. Note that $R[q] /\left(\Phi_{n}(q)\right)=$ $R \oplus R q \oplus \cdots \oplus R q^{d-1}$ with $d=\operatorname{deg} \Phi_{n}(q)$, and $\bar{a}_{l}$ is expressed as a polynomial in $q$ of degree $<d$, each coefficient of which is divisible by $p_{1} \cdots p_{k}$ in $R$ for $k \geq 0$. Since $R$ is a subring of $\overline{\mathbb{Q}}$ and each $p_{i}$ is a non-unit in $R$, it follows that the coefficients of $\bar{a}_{l}$ are zero. Consequently, we have $a_{l} \in\left(\Phi_{n}(q)\right)$.

Proposition 6.1. $\quad$ Let $R$ be a subring of $\overline{\mathbb{Q}}$, and $S \subset \mathbb{N}$ an infinite subset. Then the completion $R[q]^{S}$ of $R[q]$ is not an ideal-adic completion, i.e., there is no ideal $I$ in $R[q]$ such that $\operatorname{id}_{R[q]}$ induces an isomorphism $R[q]^{S} \simeq$ $\lim _{j} R[q] / I^{j}$.

Proof. Suppose to the contrary that there is a nonzero ideal $I$ in $R[q]$ such that $\operatorname{id}_{R[q]}$ induces an isomorphism $R[q]^{S} \simeq \varliminf_{j} R[q] / I^{j}$. Let $f(q) \in I$ be a nonzero element. Since $S$ is infinite, there is an $m \in S$ such that for each $j \geq 0$, we have $f(q)^{j} \notin \Phi_{m}(q) \overline{\mathbb{Q}}[q]$ and hence $f(q)^{j} \notin \Phi_{m}(q) R[q]$. Hence the ideals $I^{j} \subset R, j \geq 0$, are not cofinal in the ideals $(g(q)) \subset R[q], g(q) \in \Phi_{S}^{*}$. This contradicts the assumption.

Let $R$ be a subring of $\overline{\mathbb{Q}}$, and let $Z \subset Z^{\overline{\mathbb{Q}}}$ be a subset. Set

$$
P_{Z}(R)=\prod_{\zeta \in Z} R[\zeta]
$$

which generalizes the definition of $P_{Z}(\mathbb{Z})$. If $S \subset \mathbb{N}$ is a subset and $Z \subset Z_{S}^{\overline{\mathbb{Q}}}$, then let

$$
\epsilon_{S, Z}^{R}: R[q]^{S} \rightarrow P_{Z}(R)
$$

denote the homomorphism induced by $R[q] \rightarrow P_{Z}(R), f(q) \mapsto(f(\zeta))_{\zeta \in Z \text {. }}$

Theorem 6.2. $\quad$ Let $R$ be a subring of $\overline{\mathbb{Q}}$, and let $S \subset \mathbb{N}$ and $Z \subset Z_{S}^{\overline{\mathbb{Q}}}$ be subsets. Suppose that there is an element $n \in S$ such that for infinitely many 
$\zeta \in Z$ we have ord $\zeta \Leftrightarrow_{R}$ n. Then the homomorphism $\epsilon_{S, Z}^{R}: R[q]^{S} \rightarrow P_{Z}(R)$ is injective.

In particular, if $R$ is a subring of the ring of algebraic integers, and $Z \subset Z^{\overline{\mathbb{Q}}}$ is a subset containing infinitely many elements of prime power order, then $\epsilon_{S, Z}^{R}: R[q]^{S} \rightarrow P_{Z}(R)$ is injective.

Proof. Set $N_{Z}=\{\operatorname{ord} \zeta \mid \zeta \in Z\} \subset \mathbb{N}$. Let $\gamma: P_{N_{Z}}(R) \rightarrow P_{Z}(R)$ be the homomorphism defined by $\gamma\left(\left(f_{n}(q)\right)_{n \in N_{Z}}\right)=\left(f_{n_{\zeta}}(\zeta)\right)_{\zeta \in Z}$. Since $\gamma$ is the direct product of the injective homomorphisms $R[q] /\left(\Phi_{n}(q)\right) \rightarrow \prod_{\zeta \in Z \text {,ord } \zeta=n} R[\zeta]$, $f(q) \mapsto(f(\zeta))_{\zeta}$, it follows that $\gamma$ is injective. We have $\epsilon_{S, Z}^{R}=\gamma \epsilon_{S, N_{Z}}^{R}$, where $\epsilon_{S, N_{Z}}^{R}: R[q]^{S} \rightarrow P_{N_{Z}}(R)$ is injective by Theorem 6.1 . Hence $\epsilon_{S, Z}^{R}$ is injective.

Conjecture 6.1. For any infinite subset $Z \subset Z^{\overline{\mathbb{Q}}}$, the homomorphism $\epsilon_{\mathbb{N}, Z}^{\mathbb{Z}}: \mathbb{Z}[q]^{\mathbb{N}} \rightarrow P_{Z}(\mathbb{Z})$ is injective.

If $Z^{\prime} \subset Z \subset Z^{R}$, then we have a homomorphism

$$
\epsilon_{Z, Z^{\prime}}^{R}: R[q]^{Z} \rightarrow P_{Z^{\prime}}(R),
$$

induced by $R[q] \rightarrow P_{Z^{\prime}}(R), f(q) \mapsto(f(\zeta))_{\zeta}$.

Theorem 6.3. Let $R$ be a subring of $\overline{\mathbb{Q}}$, let $Z \subset Z^{R} a \Leftrightarrow_{R}$-connected subset, and let $Z^{\prime} \subset Z$. Suppose that for some $\zeta \in Z$ there are infinitely many elements $\xi \in Z^{\prime}$ with $\xi \Leftrightarrow_{R} \zeta$. Then the homomorphism $\epsilon_{Z, Z^{\prime}}^{R}: R[q]^{Z} \rightarrow P_{Z^{\prime}}(R)$ is injective.

Proof. The proof is similar to that of Theorem 6.1 with the cyclotomic polynomials replaced with the polynomials $q-\zeta$, where $\zeta$ is a root of unity. The details are left to the reader.

\section{§7. Remarks}

\section{§7.1. Units in $\mathbb{Z}[q]^{S}$}

If $R$ is a ring and $S \subset \mathcal{M}_{R}$ is a subset consisting of monic polynomials whose constant terms are units in $R$, then the element $q$ is invertible in $R[q]^{S}$. In particular, we have an explicit formula for $q^{-1} \in R[q]^{\mathbb{N}}$ as follows.

Proposition 7.1. For any ring $R$, the element $q \in R[q]^{\mathbb{N}}$ is invertible with the inverse

$$
q^{-1}=\sum_{n \geq 0} q^{n}(q)_{n},
$$

where $(q)_{n}=(1-q)\left(1-q^{2}\right) \cdots\left(1-q^{n}\right)$. 
Proof. $\quad q \sum_{n \geq 0} q^{n}(q)_{n}=\sum_{n \geq 0} q^{n+1}(q)_{n}=\sum_{n \geq 0}\left(1-\left(1-q^{n+1}\right)\right)(q)_{n}=$ $\sum_{n \geq 0}\left((q)_{n}-(q)_{n+1}\right)=(q)_{0}=1$.

For each subset $S \subset \mathbb{N}$, the inclusion $\mathbb{Z}[q] \subset \mathbb{Z}\left[q, q^{-1}\right]$ induces an isomorphism

$$
\mathbb{Z}[q]^{S} \simeq \lim _{f \in \Phi_{S}^{*}} \mathbb{Z}\left[q, q^{-1}\right] /(f),
$$

via which we will identify these two rings. If $S \neq \emptyset$, then, since $\bigcap_{f \in \Phi_{S}^{*}}(f)=$ (0) in $\mathbb{Z}\left[q, q^{-1}\right]$, the natural homomorphism $\mathbb{Z}\left[q, q^{-1}\right] \rightarrow \mathbb{Z}[q]^{S}$ is injective and regarded as inclusion.

For a ring $R$, let $U(R)$ denote the (multiplicative) group of the units in $R$. If $S \neq \emptyset$, then we have

$$
U\left(\mathbb{Z}\left[q, q^{-1}\right]\right) \subset U\left(\mathbb{Z}[q]^{\mathbb{N}}\right) .
$$

It is well known that $U\left(\mathbb{Z}\left[q, q^{-1}\right]\right)=\left\{ \pm q^{i} \mid i \in \mathbb{Z}\right\}$. If we regard $\mathbb{Z}[q]^{\mathbb{N}}$ and the $\mathbb{Z}[q]^{\langle n\rangle}$ as subrings of $\mathbb{Z}[q]^{\langle 1\rangle}=\mathbb{Z}[[q-1]]$ as in Corollary 4.1, then we have

$$
U\left(\mathbb{Z}[q]^{\mathbb{N}}\right)=\bigcap_{n \in \mathbb{N}} U\left(\mathbb{Z}[q]^{\langle n\rangle}\right) .
$$

Conjecture 7.1. We have $U\left(\mathbb{Z}[q]^{\mathbb{N}}\right)=\left\{ \pm q^{i} \mid i \in \mathbb{Z}\right\}$.

Remark 7.1. One might expect that Conjecture 7.1 would generalize to any infinite, $\mathbb{Z}$-admissible subset $S \subset \mathbb{N}$, but this is not the case. For odd $m \geq 3$, consider the element $\gamma_{m}=\sum_{i=0}^{m-1}(-1)^{i} q^{i} \in \mathbb{Z}[q]$, which is known to define a unit in the ring $\mathbb{Z}[q] /\left(q^{n}-1\right)$ with $(n, 2 m)=1$ and is called an "alternating unit", see [14]. For such $n$, it follows that there are $u, v \in \mathbb{Z}[q]$ such that $\gamma_{m} u=1+v \Phi_{n}(q)$. Since $1+v \Phi_{n}(q)$ is a unit in $\mathbb{Z}[q]^{\langle n\rangle}$, it follows that $\gamma_{m}$ is a unit in $\mathbb{Z}[q]^{\langle n\rangle}$. Set $S=\{n \in \mathbb{N} \mid(n, 2 m)=1\}$. Then it is straightforward to check that $\gamma_{m}$ defines a unit in $\mathbb{Z}[q]^{S}$ (hence also in $\mathbb{Z}[q]^{S^{\prime}}$ for any $S^{\prime} \subset S$ ). Consequently, we have $U\left(\mathbb{Z}[q]^{S}\right) \supsetneq\left\{ \pm q^{i} \mid i \in \mathbb{Z}\right\}$.

\section{$\S 7.2$. A localization of $\mathbb{Z}[q]^{\mathbb{N}}$}

In some applications, it will be natural to consider the following type of localization of $\mathbb{Z}[q]^{\mathbb{N}}$. Recall from Proposition 5.1 that $\mathbb{Z}[q]^{\mathbb{N}}$ is an integral domain. Let $Q\left(\mathbb{Z}[q]^{\mathbb{N}}\right)$ denote the quotient field of $\mathbb{Z}[q]^{\mathbb{N}}$. We will consider the $\mathbb{Z}[q]^{\mathbb{N}}$-subalgebra $\mathbb{Z}[q]^{\mathbb{N}}\left[\Phi_{\mathbb{N}}^{-1}\right]$ of $Q\left(\mathbb{Z}[q]^{\mathbb{N}}\right)$ generated by the elements $\Phi_{n}(q)^{-1}$ for 
$n \in \mathbb{N}$. Alternatively, $\mathbb{Z}[q]^{\mathbb{N}}\left[\Phi_{\mathbb{N}}^{-1}\right]$ may be defined as the subring of $Q\left(\mathbb{Z}[q]^{\mathbb{N}}\right)$ consisting of the fractions $f(q) / g(q)$ with $f(q) \in \mathbb{Z}[q]^{\mathbb{N}}$ and $g(q) \in \Phi_{\mathbb{N}}^{*}$. Similarly, let $\mathbb{Z}\left[q, q^{-1}\right]\left[\Phi_{\mathbb{N}}^{-1}\right]$ denote the $\mathbb{Z}\left[q, q^{-1}\right]$-subalgebra of the quotient field $\mathbb{Q}(q)\left(\subset Q\left(\mathbb{Z}[q]^{\mathbb{N}}\right)\right)$ of $\mathbb{Z}\left[q, q^{-1}\right]$ generated by the elements $\Phi_{n}(q)^{-1}$ for $n \in \mathbb{N}$, which may alternatively defined as the subring of $\mathbb{Q}(q)$ consisting of the fractions $f(q) / g(q)$ with $f(q) \in \mathbb{Z}\left[q, q^{-1}\right]$ and $g(q) \in \Phi_{\mathbb{N}}^{*}$.

Proposition 7.2. We have $\mathbb{Z}[q]^{\mathbb{N}}\left[\Phi_{\mathbb{N}}^{-1}\right]=\mathbb{Z}[q]^{\mathbb{N}}+\mathbb{Z}\left[q, q^{-1}\right]\left[\Phi_{\mathbb{N}}^{-1}\right]$.

Proof. The inclusion $\supset$ is obvious; we will show the other inclusion. Since

$$
\mathbb{Z}[q]^{\mathbb{N}}\left[\Phi_{\mathbb{N}}^{-1}\right]=\bigcup_{f(q) \in \Phi_{\mathbb{N}}^{*}} \frac{1}{f(q)} \mathbb{Z}[q]^{\mathbb{N}},
$$

it suffices to show that for each $f(q) \in \Phi_{\mathbb{N}}^{*}$ we have

$$
\frac{1}{f(q)} \mathbb{Z}[q]^{\mathbb{N}} \subset \mathbb{Z}[q]^{\mathbb{N}}+\frac{1}{f(q)} \mathbb{Z}\left[q, q^{-1}\right] .
$$

By multiplying $f(q)$, we need to show that

$$
\mathbb{Z}[q]^{\mathbb{N}} \subset f(q) \mathbb{Z}[q]^{\mathbb{N}}+\mathbb{Z}\left[q, q^{-1}\right],
$$

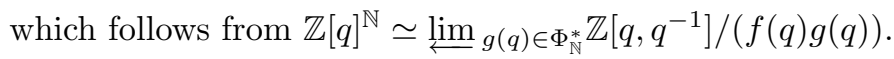

Proposition 7.3. We have

$$
\mathbb{Z}[q]^{\mathbb{N}} \cap \mathbb{Z}\left[q, q^{-1}\right]\left[\Phi_{\mathbb{N}}^{-1}\right]=\mathbb{Z}\left[q, q^{-1}\right] .
$$

Proof. The inclusion $\supset$ is obvious; we will show the other inclusion. Suppose that $f(q)=g(q) / h(q) \in \mathbb{Z}[q]^{\mathbb{N}} \cap \mathbb{Z}\left[q, q^{-1}\right]\left[\Phi_{\mathbb{N}}^{-1}\right]$, where $g(q) \in \mathbb{Z}\left[q, q^{-1}\right]$ and $h(q) \in \Phi_{\mathbb{N}}^{*}$. We may assume that $h(q)$ is minimal in degree. Thus there is no $n \in \mathbb{N}$ such that $g(q)$ and $h(q)$ have a common divisor $\Phi_{n}(q)$.

Suppose that $h(q) \neq 1$. Choose $n \in \mathbb{N}$ such that $\Phi_{n}(q) \mid h(q)$ in $\mathbb{Z}[q]$. Let $\zeta_{n} \in \overline{\mathbb{Q}}$ denote a primitive $n$th root of unity. By applying the homomorphism

$$
\sigma_{\mathbb{N},\left\{\zeta_{n}\right\}}^{\mathbb{Z}}: \mathbb{Z}[q]^{\mathbb{N}} \rightarrow \mathbb{Z}\left[\zeta_{n}\right], \quad a(q) \mapsto a\left(\zeta_{n}\right)
$$

to the both sides of the identity $g(q)=f(q) h(q)$ in $\mathbb{Z}[q]^{\mathbb{N}}$, we obtain $g\left(\zeta_{n}\right)=$ $f\left(\zeta_{n}\right) h\left(\zeta_{n}\right)=0$. Hence $g(q)$ is divisible by $\Phi_{n}(q)$ in $\mathbb{Z}\left[q, q^{-1}\right]$, which contradicts the assumption that $g(q)$ and $h(q)$ do not have a common divisor. Hence we have $h(q)=1$, and it follows that $f(q) \in \mathbb{Z}\left[q, q^{-1}\right]$. 


\section{$\S 7.3 . \quad$ Modules}

We can define cyclotomic completions also for any $\mathbb{Z}$-module, as follows. Let $A$ be a $\mathbb{Z}$-module, and let $A[q]$ denote the $\mathbb{Z}[q]$-module of polynomials in $q$ with coefficients in $A$. For each $S \subset \mathbb{N}$, let $A[q]^{S}$ denote the completion

$$
A[q]^{S}=\lim _{f \in \Phi_{S}^{*}} A[q] / f A[q]
$$

If $A$ is a ring, then this definition of $A[q]^{S}$ is compatible with the previous one. Some results in the present paper can be generalized to $A[q]^{S}$.

For example, Theorem 4.1 may be generalized as follows. Let $\Leftrightarrow_{A}$ denote the relation on $\mathbb{N}$ such that $m \Leftrightarrow_{A} n$ if and only if either we have $A=0$, or $m / n$ is an integer power of a prime $p$ such that $A$ is $p$-adically separated.

Theorem 7.1. $\quad$ Let $A$ be a $\mathbb{Z}$-module, and let $S^{\prime} \subset S \subset \mathbb{N}$ be subsets. Suppose that for each $n \in S$ there is a sequence $S^{\prime} \ni n^{\prime} \Leftrightarrow_{A} \cdots \Leftrightarrow_{A} n$ in $S$. Then the homomorphism $\rho_{S, S^{\prime}}^{A}: A[q]^{S} \rightarrow A[q]^{S^{\prime}}$ induced by $\operatorname{id}_{A[q]}$ is injective.

Proof. One way to prove Theorem 7.1 is to modify Section 3 and the proof of Theorem 4.1. We roughly sketch the necessary modifications. Section 3 is generalized as follows. For two elements $f, g \in \mathcal{M}_{R}$ and an $R$-module, we write $f \Rightarrow_{A} g$ if $f \stackrel{I}{\Rightarrow}_{A} g$ for some ideal $I$ such that $A$ is $I$-adically separated. Then Proposition 3.1 with $R$ replaced by an $R$-module $A$ holds. Generalizations of Theorem 3.1 and Corollary 3.1 to $R$-modules is straightforward. Theorem 7.1 follows immediately from the generalized version of Corollary 3.1.

Alternatively, we can use Theorem 4.1 as follows. Since the case $A=0$ is trivial, we assume not. Let $A^{\prime}=\mathbb{Z} \oplus A$ be the ring with the multiplication $(m, a)(n, b)=(m n, m b+n a)$ and with the unit $(1,0)$. Then for $m, n \in \mathbb{N}$ we have $m \Leftrightarrow_{A} n$ if and only if $m \Leftrightarrow A_{A^{\prime}} n$. Hence we can apply Theorem 4.1 to obtain the injectivity of $\rho_{S, S^{\prime}}^{A^{\prime}}$. We can identify $\rho_{S, S^{\prime}}^{A^{\prime}}$ with the direct product

$$
\rho_{S, S^{\prime}}^{\mathbb{Z}} \oplus \rho_{S, S^{\prime}}^{A}: \mathbb{Z}[q]^{S} \oplus A[q]^{S} \rightarrow \mathbb{Z}[q]^{S^{\prime}} \oplus A[q]^{S^{\prime}} .
$$

Hence $\rho_{S, S^{\prime}}^{A}$ is injective.

\section{$\S 7.4$. Non-surjectivity of $\rho_{\mathbb{N},\{n\}}^{\mathbb{Z}}$}

Proposition 7.4. We have the following.

1. If $m, n \in \mathbb{N}, m \Leftrightarrow_{\mathbb{Z}} n$, and $m \neq n$, then the homomorphism $\rho_{\{m, n\},\{m\}}^{\mathbb{Z}}$ is not surjective. 
2. If $m \mid n$ and $m \neq n$, then the homomorphism $\rho_{\langle n\rangle,\langle m\rangle}^{\mathbb{Z}}$ is not surjective.

3. For each nonempty, finite subset $S \subset \mathbb{N}$, the homomorphism $\rho_{\mathbb{N}, S}^{\mathbb{Z}}$ is not surjective.

Proof. (1) We have $m / n=p^{e}$ for some prime $p$ and an integer $e \neq 0$. Consider the following commutative diagram of natural homomorphisms.

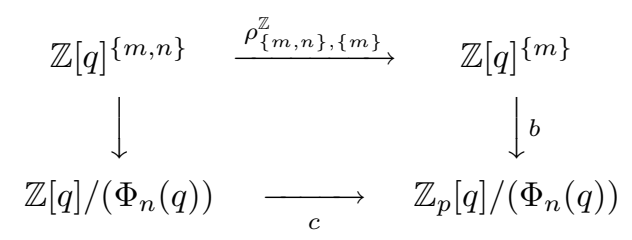

It follows from $\mathbb{Z}_{p}[q] /\left(\Phi_{n}(q)\right) \simeq \varliminf_{j} \mathbb{Z}[q] /\left(\Phi_{n}(q), p^{j}\right), \Phi_{m}(q) \in \sqrt{\left(\Phi_{n}(q), p\right)}$, and $p \in\left(\Phi_{m}(q), \Phi_{n}(q)\right)$ (which follows from (4.2)) that $b$ is a well-defined, surjective homomorphism. Since $c$ is not surjective, $\rho_{\{m, n\},\{m\}}^{\mathbb{Z}}$ is not surjective.

(2) We may assume that $n=p m$ for a prime $p$. The case $m=1$ is contained in (1) above. There are isomorphisms $\mathbb{Z}[q]^{\langle m\rangle} \simeq \mathbb{Z}\left[q^{m}\right]^{\langle 1\rangle} \otimes_{\mathbb{Z}\left[q^{m}\right]}$ $\mathbb{Z}[q]$ and $\mathbb{Z}[q]^{\langle p m\rangle} \simeq \mathbb{Z}\left[q^{m}\right]^{\langle p\rangle} \otimes_{\mathbb{Z}\left[q^{m}\right]} \mathbb{Z}[q]$ induced by the isomorphism $\mathbb{Z}[q] \simeq$ $\mathbb{Z}\left[q^{m}\right] \otimes_{\mathbb{Z}\left[q^{m}\right]} \mathbb{Z}[q]$. Thus the case $m=1$ implies the non-surjectivity of $\rho_{\langle p m\rangle,\langle m\rangle}^{\mathbb{Z}}$.

(3) The homomorphism $\rho_{\mathbb{N}, S}^{\mathbb{Z}}$ factors as follows.

$$
\mathbb{Z}[q]^{\mathbb{N}} \stackrel{\rho_{\mathbb{N},\langle n\rangle}^{\mathbb{Z}}}{\longrightarrow} \mathbb{Z}[q]^{\langle n\rangle} \stackrel{\rho_{\langle n\rangle,\langle m\rangle}^{\mathbb{Z}}}{\longrightarrow} \mathbb{Z}[q]^{\langle m\rangle} \stackrel{\rho_{\langle m\rangle, S}^{\mathbb{Z}}}{\longrightarrow} \mathbb{Z}[q]^{S}
$$

where $m \in \mathbb{N}$ is the least common multiple of the elements of $S$, and $n \in \mathbb{N}$ is any element such that $m \mid n$ and $m \neq n$. By (2) above, $\rho_{\langle n\rangle,\langle m\rangle}^{\mathbb{Z}}$ is not surjective.

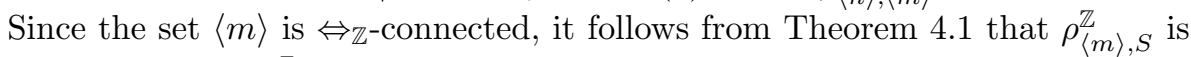
injective. Hence $\rho_{\mathbb{N}, S}^{\mathbb{Z}}$ is not surjective.

\section{$\S 7.5$. The ring $\mathbb{Q}[q]^{S}$}

The structure of $\mathbb{Q}[q]^{S}$ for $S \subset \mathbb{N}$ is quite contrasting to that of $\mathbb{Z}[q]^{S}$. Note that $\mathbb{Z}[q]^{S}$ embeds into $\mathbb{Q}[q]^{S}$ by Lemma 3.1. (The following remarks holds if we replace $\mathbb{Q}$ with any ring $R$ such that each element of $S$ is a unit in $R$.)

Note that if $m, n \in S, m \neq n$, then $\left(\Phi_{m}(q)^{i}, \Phi_{n}(q)^{j}\right)=(1)$ in $\mathbb{Q}[q]$ for any $i, j \geq 0$. Consequently, for each $f(q)=\prod_{n \in S} \Phi_{n}(q)^{\lambda(n)} \in \Phi_{S}^{*}$ with $\lambda(n) \geq 0$ we have by the Chinese Remainder Theorem

$$
\mathbb{Q}[q] /(f(q)) \simeq \prod_{n \in S} \mathbb{Q}[q] /\left(\Phi_{n}(q)^{\lambda(n)}\right) .
$$


Taking the inverse limit, we obtain an isomorphism

$$
\mathbb{Q}[q]^{S \stackrel{\simeq}{\longrightarrow}} \prod_{n \in S} \mathbb{Q}[q]^{\{n\}} .
$$

Since each $\mathbb{Q}[q]^{\{n\}}$ is not zero, it follows that $\mathbb{Q}[q]^{S}$ is not an integral domain if $|S|>1$. It also follows that $\rho_{S, S^{\prime}}^{\mathbb{Q}}: \mathbb{Q}[q]^{S} \rightarrow \mathbb{Q}[q]^{S^{\prime}}$ is not injective (but surjective) for each $S^{\prime} \subsetneq S$. Since for each $n \in S$ the (surjective) homomorphism $\mathbb{Q}[q]^{\{n\}} \rightarrow \mathbb{Q}[q] /\left(\Phi_{n}(q)\right)$ is not injective, the homomorphism $\epsilon_{S, S}^{\mathbb{Q}}: \mathbb{Q}[q]^{S} \rightarrow P_{S}(\mathbb{Q})$ is not injective.

\section{Acknowledgements}

The author would like to thank the anonymous referee for helpful comments.

\section{References}

[1] Habiro, K., On the quantum $s l_{2}$ invariants of knots and integral homology spheres, Invariants of knots and 3-manifolds (Kyoto, 2001), 55-68 Geom. Topol. Monogr., 4, Geom. Topol. Publ., Coventry, 2002.

[2] $\_$, An integral form of the quantized enveloping algebra of $s l_{2}$ and its completions, Preprint.

[3]

[4] Lawrence, R. J., Asymptotic expansions of Witten-Reshetikhin-Turaev invariants for some simple 3-manifolds, J. Math. Phys., 36 (1995), 6106-6129.

[5] Witten-Reshetikhin-Turaev invariants of 3-manifolds as holomorphic functions, Geometry and physics (Aarhus, 1995), 363-377, Lecture Notes in Pure and Appl. Math., 184, Dekker, New York, 1997.

[6] Lawrence, R. J. and Zagier, D., Modular forms and quantum invariants of 3-manifolds, Asian J. Math., 3 (1999), 93-107.

[7] Le, T. T. Q., Quantum invariants of 3-manifolds: integrality, splitting, and perturbative expansion, Topology Appl., 127 (2003), 125-152.

[8] Matsumura, H., Commutative ring theory, Cambridge Studies in Advanced Mathematics, 8, Cambridge University Press, Cambridge, 1986.

[9] Murakami, H., Quantum SU(2)-invariants dominate Casson's SU(2)-invariant, Math. Proc. Cambridge Philos. Soc., 115 (1994), 253-281.

[10] Ohtsuki, T., A polynomial invariant of integral homology 3-spheres, Math. Proc. Cambridge Philos. Soc., 117 (1995), 83-112.

[11] Ohtsuki, T. (ed.), Problems on invariants of knots and 3-manifolds, Invariants of knots and 3-manifolds (Kyoto, 2001), 377-572 Geom. Topol. Monogr., 4, Geom. Topol. Publ., Coventry, 2002.

[12] Reshetikhin, N. and Turaev, V. G., Invariants of 3-manifolds via link polynomials and quantum groups, Invent. Math., 103 (1991), 547-597.

[13] Rozansky, L., On $p$-adic properties of the Witten-Reshetikhin-Turaev invariant, Preprint, math.QA/9806075.

[14] Sehgal, S. K., Units in integral group rings, Pitman Monogr. Surveys Pure Appl. Math., Longman, Essex, 1993.

[15] Witten, E., Quantum field theory and the Jones polynomial, Comm. Math. Phys., 121 (1989), 351-399.

[16] Zagier, D., Vassiliev invariants and a strange identity related to the Dedekind etafunction, Topology, 40 (2001), 945-960. 